\title{
Jakob Klaesi - Schlafkur und Antieidodiathese
}

Von Thomas Haenel

\section{Einleitung}

Wenn der Person und des Wirkens Jakob Klaesis gedacht wird, dann geschieht dies nicht wegen des patriarchalischen Alters - Klaesi, Nestor der Schweizer Psychiatrie, konnte in diesem Jahr seinen 96. Geburtstag feiern -, sondern es geschieht deshalb, weil der heutigen Ärztegeneration ein einst international bekannter Psychiater in Erinnerung gebracht oder vorgestellt werden soll. Er ist einer jener Ärzte, die während Jahrzehnten die schweizerische Psychiatrie wesentlich beeinflußt und auch zu aktuellen, nichtpsychiatrischen Fragen Stellung bezogen haben. Viele seiner Äußerungen und Ansichten sind im Spiegel seiner Zeit zu sehen oder zu interpretieren; mindestens ebenso viele sind aber heute genauso aktuell oder sogar noch aktueller als damals. Seine Originalität, seine humorvollen, oft ironischen und träfen Formulierungen, mit denen er manche «Moden» oder Einseitigkeiten brandmarkte, sind zeitlos.

Bei meinen Besuchen auf Schloß Knonau in den Jahren 1975 und 1979 trat ich einem großgewachsenen, ernsten, aber doch freundlichen «Schloßherrn» gegenüber. Als ich ihm im prächtig ausgestatteten Salon helfen wollte, in einem Sessel Platz zu nehmen, schaute er mich an und meinte fast vorwurfsvoll, so etwas könne er noch alleine! Sogleich begann Klaesi das Gespräch: er fragte, ob ich wüßte, was (Anti)-eidodiathese sei? (Ich wußte es bei meinem ersten Besuch noch nicht!) Er begann, von seiner internationalen Praxis zu berichten, von Patienten, deren Leiden er in ein oder zwei Sitzungen zu lindern oder zu heilen vermochte mit seiner Art Psychotherapie, die den Menschen als Ganzes erfaßt. Klaesi machte kein Hehl daraus, daß er von Therapien, die jahrelang dauern und sich auf die Psychoanalyse gründen, nichts halte. Die Zeit verging im Fluge. Ich mußte mich nach dem Kaffee wieder auf den Weg zum Bahnhof machen. Klaesi begleitete mich noch bis zum Ausgang. Zuvor jedoch mußten wir an einem riesigen Hund vorbei, der das Schloß bewacht. Die erlebten Momente bleiben mir unvergeßlich, Erinnerungen an eine Begegnung, bei der in einem alten, aristokratischen Herrn Schweizer Psychiatriegeschichte auflebte und lebendig wurde.

\section{Werdegang}

Klaesi wurde am 29. Mai 1883 geboren und wuchs im Kanton Glarus als Sohn eines Kaufmanns auf. Er stammt aus einer sehr alten Schweizer Familie. Sie ist seit dem 14. Jahrhundert im Kanton Glarus bekannt ${ }^{28}$.

Klaesi hat die große Schulstube in Nidfurn (Kt. Glarus) mit 40-50 Schulkindern aus sieben verschiedenen Klassen in bester Erinnerung. Hausaufgaben gab es damals, in ländlichen Verhältnissen, keine. Trotzdem waren die Schüler, wenn sie am Ende der sechsten Klasse 
die Aufnahmeprüfung in die Sekundarschule bestehen mußten, nicht schlechter als andere. Seinem Lehrer, Joachim Blumer, widmete Klaesi später einige Verse. Lobend äußert sich Klaesi über die Aarauer Kantonsschule, die wegen der ausgezeichneten Lehrer und der herrschenden Strenge bestens bekannt gewesen sein soll ${ }^{27}$.

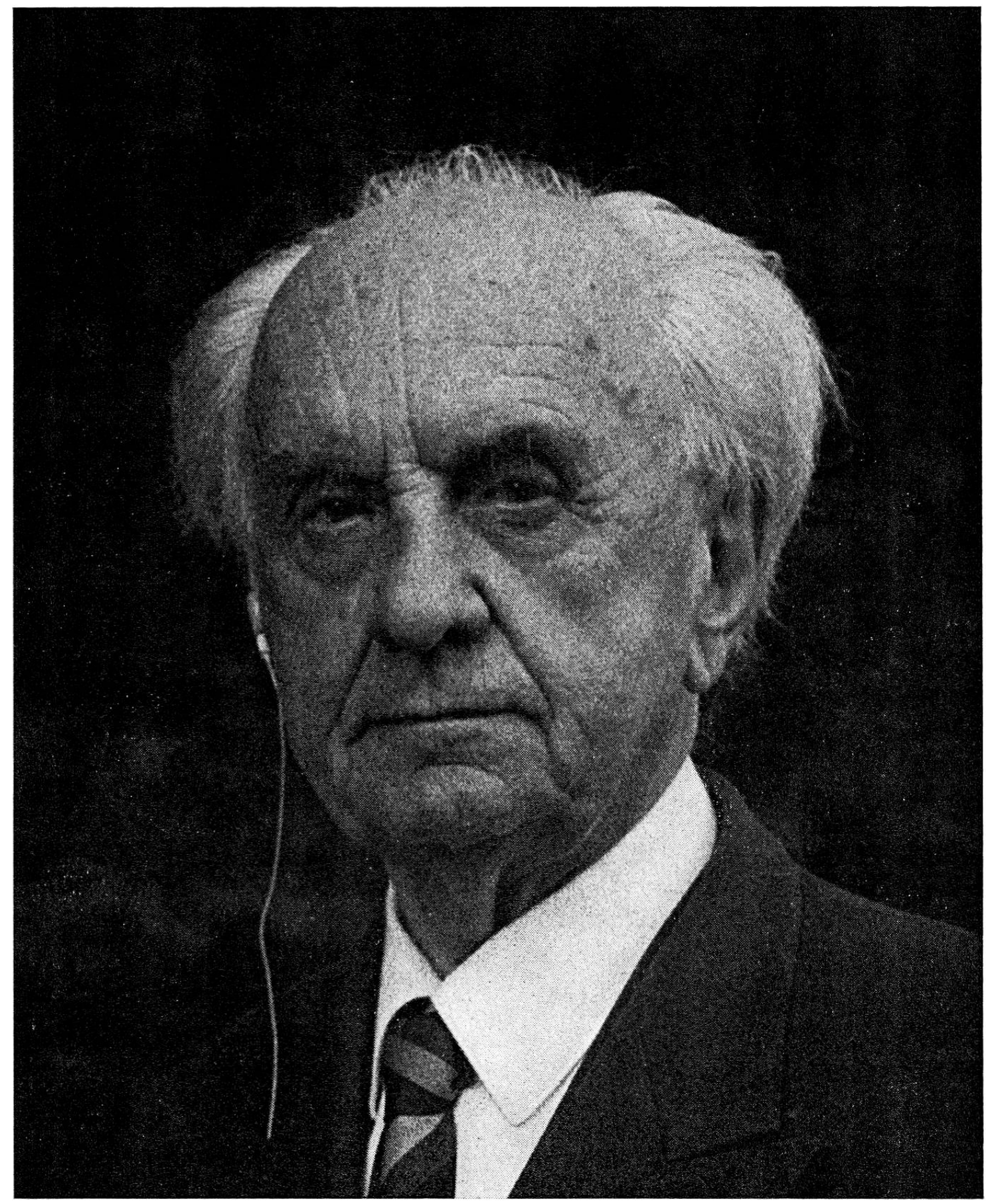

Jakob Klaesi 
Schon als Kind hatte Klaesi den Wunsch, Arzt zu werden, um einen Fall von manischdepressiver Psychose im engsten Verwandtenkreis heilen zu können. Er wäre aber auch gerne Schriftsteller oder Schauspieler geworden. Die Eltern bestanden jedoch darauf, daß ihr Sohn einen Beruf mit einem gesicherten Einkommen ergreife.

1903 immatrikulierte sich Klaesi an der Medizinischen Fakultät der Universität Zürich. Klinische Auslandsemester führten ihn nach Kiel und München. In München war er von den beiden weltberühmten Lehrern Friedrich von Müller und Emil Kraepelin tief beeindruckt. Sie wurden Wegweiser für seine psychiatrische Zukunft $^{27}$. Klaesi sah, wie Friedrich von Müller eine hysterische Aphonie zufällig durch Kehlkopfspiegeln heilte, weil die Patientin der Ansicht war, der Spiegel sei zu therapeutischen Zwecken in ihren Mund eingeführt worden. Der Professor verzichtete jedoch auf billigen Ruhm und verordnete der Kranken sofort vollständige Ruhe und Schweigen bis zur nächsten Arztvisite. Den Studenten erklärte er, daß der Patientin sonst auf der Abteilung vorgehalten würde, ihre Krankheit sei nur Einbildung gewesen ${ }^{10}$. An diesen Fall anknüpfend, fährt Klaesi fort: "Also legt man ihr jetzt eine Binde um den Mund. Sie darf nur noch mit uns Ärzten sprechen, bekommt eine strenge Schonkost und darf keine Besuche empfangen. Nach 3, 4 Wochen daheim, wissen dann alle, sie hatte einen mehrwöchigen Klinikaufenthalt nötig gehabt. Was wir sie fragen: 'Ob und warum ihr Wort keinen Widerhall mehr fand, sich nicht lohnte, oder was sie fürchtete, gefragt $\mathrm{zu}$ werden und nicht preisgeben wollte.'» ${ }^{27,10}$ Es wurde später zu einem Prinzip Klaesis, solchen Kranken den Rückzug in das Gesundsein zu decken.

Klaesi erlebte Kraepelin als großzügigen, ernsten und gütigen Dozenten mit einem ausgezeichneten Gedächtnis. In München gab es schon damals eine lange Praktikantenliste, welche die Namen der Studenten enthielt, die für die Vorlesung zum Praktizieren aufgerufen wurden. Klaesi kam gedankenlos der Bitte zweier Kollegen nach, an ihrer Stelle praktizieren zu gehen, wenn sie aufgerufen würden. Als Klaesi selbst an die Reihe kam und seine beiden Mitpraktikanten die Frage über einen Quartalssäufer nicht beantworten konnten, wandte sich Kraepelin Klaesi zu mit den Worten: "Sie sind also heute in eigener Mission hier, Herr Klaesi? $»^{27}$

In jener Zeit mußte Klaesi mit einem schwerwiegenden persönlichen Problem fertig werden. Er bemerkte, daß er anläßlich eines Opernbesuches zwar noch Töne, aber nicht mehr die Worte verstehen konnte. Ein peinigendes, Tag und Nacht währendes Ohrensausen gesellte sich dazu. Nach gründlichen otologischen Abklärungen im In- und Ausland wurde Klaesi in Aussicht gestellt, aufgrund seiner beginnenden Otosklerose mit 30 Jahren völlig taub zu sein. Trotzdem ließ er sich nicht entmutigen: Er blieb Reiter, Bergsteiger und ließ sich nicht einmal 
aus der Armee ausmustern. Er wußte auch, daß jede chronische Krankheit jederzeit zum Stillstand kommen kann ${ }^{27}$. Die Zukunft gab Klaesi recht: er blieb zwar schwerhörig, wurde aber nie völlig taub.

Klaesi absolvierte seine letzten Semester wieder in Zürich und schloß hier auch sein Studium 1909 ab. Nach einer chirurgischen Assistententätigkeit in Frauenfeld wurde er Assistent bei Eugen Bleuler in Zürich ${ }^{27}$. Er machte sich sogleich an seine Dissertation «Über das psychogalvanische Phänomen». ${ }^{8}$

1913 rückte Klaesi zum ersten Assistenzarzt im Burghölzli auf, und 1915 übernahm er die von Bleuler neugeschaffene Oberarztstelle. Er mußte den ersten Oberarzt, H.W.Maier, der während des Krieges lange abwesend war, vertreten, die psychiatrische Poliklinik leiten und Vorlesungen halten. Die Vorlesungen wurden von seinem Chef, Bleuler, mit dem er während seines ganzen Lebens befreundet war, regelmäßig besucht. Bleuler billigte es, daß Klaesi in der Deutung und der Therapie der Neurosen eigene Wege ging und von der Freudschen und Jungschen Schule abwich ${ }^{27}$.

Klaesi überraschte immer wieder durch unkonventionelle und originelle Fragen oder Äußerungen. Schon als 30 jähriger sagte er in seiner Sitzung der Vereinigung Südwestdeutscher Nerven- und Irrenärzte: «Die Irrtümer, welche die Psychiater seit eh und je in ihren Anstalten begangen haben und noch begehen, sind wohl folgenschwerer und zahlreicher als die ihrer Geisteskranken.» Er löste damals nicht nur Heiterkeit, sondern auch Widerspruch und Scharren aus ${ }^{27}$.

1920 erschien Klaesis Habilitationsarbeit «Über das Wesen und die Bedeutung der Stereotypien ». ${ }^{11}$ Stereotypien waren für Klaesi «längst nicht mehr nur Füllsel, Wortscherben, Bewegungsstummel, Nachäffereien, sondern 'Anrufe - Fingerzeige - Einladungen an den Berufenen, zu verstehen, Türen zu öffnen' ». ${ }^{27}$

Von 1923 bis 1926 war Klaesi als Oberarzt der Friedmatt und Leiter der neueröffneten Psychiatrischen Poliklinik in Basel tätig ${ }^{7}$. Danach eröffnete er eine Privatklinik auf Schloß Knonau (Kanton Zürich); 1933 wurde er als Direktor und Ordinarius für Psychiatrie an die Waldau nach Bern gewählt. Bis in seine hohen Siebzigerjahre behandelte Klaesi, auch nach seinem Rücktritt von der Psychiatrischen Universitätsklinik Bern, noch Patienten in seiner Privatklinik in Knonau. 1939-1941 war Klaesi Dekan der Medizinischen Fakultät Bern, 1950-1951 Rektor der Universität Bern.

\section{Klaesi in Basel}

Während seiner Basler Zeit hat Klaesi die Arbeitstherapie zwar nicht eingeführt, ihr aber neue Impulse verliehen: «... Ich habe in den Jahren 1923 und 1924 als 
Oberarzt der baselstädtischen Heil- und Pflegeanstalt Friedmatt bei Einführung einer etwas ausgiebigeren Arbeitstherapie in allererster Linie die Anschaffung einer Reihe von Spielen befürwortet und das Pflegepersonal angewiesen, bettlägerige, abweisende und faule Kranke soviel zum Spielen heranzuziehen, bis sie anfingen, sich für die Zeit und Mühe, die man für sie verwendete, dankbar zu zeigen; einmal so weit, sollte die Pflegeperson, die sich mit ihnen abgegeben hatte und ihnen nun lieb geworden war, um eine kleine Handreichung bitten. Gedacht, getan; ehe sie sich's versahen, waren dieser und jener bei denjenigen, die am fleißigsten mithalfen. ${ }^{18}$

Bei der Arbeitstherapie ging es Klaesi nicht darum, die Kranken zu zerstreuen, sondern vielmehr darum, ihr Selbstvertrauen und ihre Zukunftshoffnung zu stärken ${ }^{10}$. In der Arbeitstherapie sieht er keinen haushälterischen Gesichtspunkt, sondern einen rein menschlichen ${ }^{18}$. Konsequent wendet sich Klaesi deshalb auch gegen die damals zunehmende Gepflogenheit jüngerer praktizierender Ärzte, seelisch Kranken absolute Ruhe und Enthaltung von der Arbeit zu verordnen. Er fährt fort: «Gefördert wird diese immer mehr um sich greifende Erziehung zur Faulheit merkwürdigerweise am meisten durch diejenigen Institute, welche das größte Interesse daran haben sollten, die Leute tüchtig und schaffensfroh und gegen Bagatellen von Krankheit und Verletzungen gleichgültig zu erhalten, nämlich die Krankenkassen. „ ${ }^{10}$ Klaesi setzte sich auch für die Frühentlassungen der Kranken ein; dieses Prinzip fand auch später in der Waldau Anwendung ${ }^{31}$.

Klaesi dozierte während seiner Basler Zeit in Zürich. Jede Woche fuhr er mit der Eisenbahn mit einer Anzahl Basler Patienten - oft waren es Kinder - nach Zürich und stellte sie dort den Studenten vor ${ }^{28}$.

\section{Schlafkur und somatische Behandlungsmethoden}

Klaesi ging als Entdecker der sogenannten Schlafkur mit Somnifen* in die Psychiatriegeschichte ein. Es ist ein Paradoxon, daß Klaesi 1921 für eine somatische Behandlungsmethode bekannt wurde, obschon er in erster Linie Psychotherapeut war. Die Schlafkur mit Somnifen, einem Barbiturat, war für ihn nur Mittel zum Zweck ${ }^{12,13}$. Er hatte im Burghölzli eine schizophrene Patientin zu behandeln, an die man wegen ihrer Aggressivität gar nicht herankommen konnte. Ihm kam die Idee, die Patientin abhängig und hilfsbedürftig werden zu lassen, um sie auf diese Weise einer Zuwendung und Behandlung gefügiger zu machen.

\footnotetext{
* Somnifen ist eine wässerige Lösung von diäthyldipropenylbarbitursaurem Diäthylamin; das Präparat, das mit Veronal und Luminal verwandt ist, wurde von der Firma Hoffmann-La Roche hergestelltt ${ }^{12,13}$.
} 
Er versuchte es mit einer Schlafkur mit Somnifen. Eine solche dauerte etwa eine Woche und gelangte bei Erregungszuständen und halluzinatorischen Schüben zur Anwendung. Klaesi hielt Somnifen nicht für ein Wundermittel, sondern für die Voraussetzung zu einer Psychotherapie.

Klaesi wendet sich gegen die kritiklose Anwendung der sog. somatischen Behandlungsmethoden, die er mit chirurgischen Methoden vergleicht: «Kommt hinzu die offenbar schon immer und auch damals bestanden habende 'Neurose' der Psychotherapeuten und Psychiater, die aus quälender Befürchtung, ihre Behandlungsmethoden seien in den Augen der Öffentlichkeit denen der Internisten oder gar Chirurgen nicht gleichwertig, jede Gelegenheit zu körperlichen Anwendungen und Prozeduren gierig aufgreifen, verallgemeinern und zu Tode reiten, ganz besonders, wenn dazu Instrumente nötig sind, und die Kur an Eindrücklichkeit einem chirurgischen Eingriff nicht nachsteht. Man denke an die Elektroschockbehandlung, die sogar in unserer pädagogisch, psychologisch und psychoanalytisch viel interessierten Schweiz neulich sogar gegen Begehrungsvorstellungen und neurotische Symptome allerart angewendet werden wollte und noch angewendet wird. ${ }^{23}$

Im Zusammenhang mit der Elektroschockbehandlung schreibt Klaesi, nachdem er davor gewarnt hat, sie, gleichsam als therapeutischen Reflex, gegen alle Formen von nervösen Beschwerden oder psychischen Verstimmungen anzuwenden: «Geht aber der Unfug der Verallgemeinerung der Methode weiter, so ist zu erwarten, daß in Kürze oder Bälde die Elektroschockapparate wie Staubsauger mit Gebrauchsanweisung zur Selbstbedienung ins Haus geliefert werden müssen. ${ }^{21}$

In seinem Artikel «Ohne Insulin» betont Klaesi, daß die wichtigste aller Heilmaßnahmen bei Schizophrenen immer noch die psychische Behandlung sei. Dauerschlaf, Cardiazol oder Insulinbehandlung seien nur Hilfen. Klaesi wendet sich gegen die Forderung, die von gewissen Kreisen erhoben wurde, jeden neu aufgenommenen Patienten in einer psychiatrischen Anstalt einer Insulinkur zu unterziehen ${ }^{20}$.

\section{Ansichten zur Neurosenlehre}

Klaesis Anschauungen über Neurosen decken sich zu einem Teil mit denen Hans Binders. Er zitiert in seiner «Neurosenlehre» Binder, der die Neurose wie folgt definiert: «Eine Neurose ist dann zu diagnostizieren, wenn bei negativem körperlichem Befund Störungen im vegetativen Nervensystem oder bestimmte psychische Symptome ... auftreten, die in übermäßiger Intensität und zäher Fixa- 
tion sich immer wieder bemerkbar machen und vom Patienten selbst als fremdartig und störend erlebt werden, weil er seine eigenen Symptome nicht versteht.» Für Klaesi ruft nicht die Verdrängung die Neurose hervor, sondern die unvollständige Verdrängung ${ }^{24}$. Dem Neurotiker, dem «Unvollständig-Verdrängenden », fällt immer wieder Konfliktmaterial ein und nötigt ihn zur Einsicht: «Es ist und ist nicht, fehlt dir und fehlt dir nicht. ${ }^{24}$ Diese Ambivalenz unterscheidet sich von der des Schizophrenen. Die neurotische Ambivalenz wird von Klaesi mit folgenden Worten wiedergegeben: «Darum die schaukelnde Gewißheit, das zagende Besserwissen, die wankelmütige Voreingenommenheit, hoffende Angst, flehende Scheu, die Widerstreite, die den auf erlösende Antwort Brennenden unermüdlich von sich und seiner Krankheit reden machen und so dem Schein lästigster Egozentrität aussetzen, die Selbstverkleinerungen und -herabsetzungen, die mit aufwiegendem Aufbruch und Gehaben überklebt und übertrumpft werden müssen, Brüchigkeiten der Einstellung und des Vorhabens, Uneinheitlichkeit und Abschüssigkeit der Grundstimmung, die sich in Disharmonien der Architektur der Ausdrucksbewegungen, in Fehlleistungen (Verlesen, Versprechen, Verhören, Verschreiben, Straucheln, Verunfallen) und Zwangsantrieben kundtun - alles zusammen ein Ausdruckshader, der im neurotischen Syndrom auch des beherrschtesten und scheinbar gleichgültigsten Leidenden nicht fehlt und darum differentialdiagnostisch führend ist. ${ }^{24}$

Mit Recht, und für die damalige Zeit keineswegs selbstverständlich, betont Klaesi die Einheit zwischen Körperlichem und Seelischem. Die Affekte teilen sich in den sogenannten Ausdrucksphänomenen, wie z. B. Mimik, Gebärde, Gang, Haltung, Sprache usw. mit ${ }^{24}$. Klaesi führt in seiner Neurosenlehre verschiedene neue Begriffe ein. Er spricht z. B. nicht vom «sozialen Trieb», sondern er teilt alle altruistischen Triebregungen in die «aristophoren» und die «kratophoren » ein (Aristos: der Beste, Edelste; Kratos: Stärke, Gewalt). Unter ersteren versteht Klaesi diejenigen Triebregungen und Triebhandlungen, die den Drang zu Hingabe und Opfer oder auch nur ein Anschluß- und Einordnungsbedürfnis darstellen; unter letzteren versteht er diejenigen Triebregungen und Handlungen, welche der Genuß- und Lustbeschaffung dienen. Der neurotische Konflikt besteht im wesentlichen «in gegenseitigen Hemmungen und Abriegelungen von kratophoren und aristophoren Triebregungen und Willenshandlungen ...» ${ }^{24}$ Der Begriff der aristophoren Triebregungen, bzw. -handlungen, ist weiter gefaßt als der des «sozialen Triebes», aber enger als der Freudsche Begriff «Libido». Die kratophoren Triebregungen dienen der Befriedigung der Ichtriebe (Nahrungs-, Geschlechts-, Geltungstrieb) ${ }^{19}$. Was der Neurotiker auch immer tut, hält er in der Regel für falsch. «Eine eigentliche 'Lösung' wie die, daß er gleichzeitig aristophor 
und kratophor handelt, seine Vorteile sucht und damit auch der Allgemeinheit nützt und umgekehrt, gelingt ihm erst recht selten oder gar nicht. » ${ }^{19}$

Für Klaesi ist das Ziel allen Handelns und Wollens die Selbstverwirklichung. Er versteht darunter Teilhaberschaft und Teilsein, wobei die Gemeinschaft, in der man verwirklicht, raum- und zeitlos ist. Die Selbsterkenntnis ist die Folge der «Einkehr in den Geist und zum Beheimatetsein-in-ihm». ${ }^{24}$ Dieser Selbsterkenntnis folgt das Erkennen, so zu sein, wie man ist. In der Praxis heißt dies z. B.: «Der Arglose klagt sich nicht mehr an, daß er getäuscht wird, denn er hat erkannt, daß ihm Mißtrauen Klage wäre und Verfinsterung; der Gütige findet sich damit ab, daß man ihn ausbeutet, denn Schonung des Besitzes ist ihm unnatürlich und Verarmung, und der sich nicht bequem einzurichten versteht, sondern sich müht und abrackert, erfährt im Feierabend, daß Liebe, gleichgültig wem sie gilt, Tieren, Boden oder Menschen, das einzige Gute ist, das wächst, indem man es verschwendet. ${ }^{24}$

\section{Klaesi und die Lehren Freuds}

Klaesi stand den Lehren Freuds kritisch gegenüber; er akzeptierte sie nicht alle.

«Zu Polemiken können die Vergleiche mit der Psychoanalyse führen, wenn es heißt, jene baue auf das Ich-Es-Erlebnis, während die 'Médecine de la personne' auf das Ich-Du-Erlebnis gründe, oder die Psychoanalyse Freuds habe die Verbindung nach unten wieder hergestellt, indessen es jetzt gelte, die Beziehung nach oben, zum Geistigen, zu schaffen, denn die Psychoanalyse nennt als sehr wesentliches Heilungsmoment die Übertragung auf den Arzt, also auch ein IchDu-Erlebnis, und ihr Begriff der Sublimation rührt auch an Geistiges. Einer ihrer frühesten und namhaftesten Vertreter, Alphonse Mäder, hat auch schon vor 40 Jahren auf die 'Synthese' hingewiesen, die sich der Analyse anzuschließen habe, wobei er bald der Stellung im Glauben einen ersten Rang anwies. Zudem ist es für das psychoanalytische Schrifttum charakteristisch, daß alles irgendwann, irgendwo, irgendwie einmal gesagt ist, und wenn nicht gesagt, doch angedeutet oder zwischen die Zeilen gelegt. Klar und unwiderleglich ist nur die Tatsache, daß die Psychoanalyse auf der irrigen Auffassung Freuds aufgebaut hat, der Mensch denke in Worten und in einer Sprache, ein Irrtum, der sich in seiner psychoanalytischen Chiffriermethode und in der Traumdeutung katastrophal auswirkt ...» ${ }^{24}$

Träumen mißt Klaesi, wie Freud, eine wesentliche Bedeutung bei, interpretiert sie jedoch grundsätzlich anders als Freud, der Begründer der Psychoanalyse: «Die Beherrschung der Traumdeutung ist einer der wichtigsten und unerläßlich- 
sten Ausrüstungsgegenstände des Arztes, besonders des praktischen Arztes. Nach dem, was wir ... über die Chiffriermethode Freuds gesagt haben, zerlegt der Ausdrucksphänomenologe auch den Traum nicht in seine einzelnen Elemente, um dann zu jedem derselben Einfälle zu sammeln und aus diesen die Deutung abzuleiten, sondern er nimmt den Traum als Ganzes, als ein Geschehen mit einem ganz bestimmten Affektgehalt, der zur Frage überleitet, warum Angst, warum Lebensüberdruß, warum Rache usw. und dann als Gleichnis ... ${ }^{24}$

Klaesi glaubt nicht an die Traumzensur im Sinne von Sigmund Freud ${ }^{6}$. Das, was so aussehe, sei in Tat und Wahrheit Ambivalenz. Auch im Bewußtsein wisse der Mensch nicht immer, ob und warum er etwas wolle, und diese Ambivalenz nehme er gleichsam mit in den Schlaf, bzw. in den Traum. Auch am Tag werde oft etwas verblümt gesagt oder durch einen Witz angedeutet. «Wer einheitlich will oder sagen darf, was er will oder zum Verbrämen zu dumm ist, macht auch im Traum wenig Umschweife, z.B. entschlossene Brautleute, das Kind und der Oligophrene. ${ }^{24}$

\section{Psychotherapie}

Klaesi kümmerte sich nicht nur um die «lohnenden» oder «aussichtsreichen » Patienten, sondern auch um «hoffnungslose». In den dreißiger Jahren schrieb er: «2/3 aller Kranken, die bei uns jahraus, jahrein Hilfe suchen, in unseren Anstalten aufgenommen werden, können nach kürzerer oder längerer Zeit gebessert oder geheilt entlassen werden; aber das dritte Drittel bleibt zurück und wird zu unserem dauernden Bestand. Sie denken, daß an ihnen alle ärztliche Anstrengung zuschanden werde, und für sie alle Mühe umsonst sei. Ich behaupte aber, daß gerade sie es sind, die dem Arzt zu höchster Genugtuung verhelfen und seinen Beruf verklären, sind es doch diejenigen, die ohne ihn nicht leben können. „ ${ }^{18}$ Konsequent wendet sich Klaesi gegen eine Aufteilung der psychiatrischen Klinik in ein Spital für Akut-Kranke und in ein solches für Chronisch-Kranke, da letztere "von allerhöchstem Wert» seien und zu einer psychiatrischen Klinik gehörten. Die chronischen Zustandsbilder verhalten sich zu den akuten Stadien laut Klaesi - «wie die gesammelten Schriften eines Politikers zu seiner Jungfernrede im Nationalrat». ${ }^{16}$

In seiner Rektoratsrede vom 18. November 1950 definierte Klaesi den unheilbar Kranken wie folgt: «Von Unheilbarkeit eines Kranken rede ich dann, wenn er infolge seiner physischen und psychischen Ausfälle und Mängel dauernd außerstande bleibt, seine Persönlichkeit zu verwirklichen. ${ }^{22}$ In der gleichen Rede bemerkt er: «Das alles ist der Arzt: ein Wissenschaftler, ein Krieger, ein Erbar- 
mer, ein Priester und ein Künstler. Sein höchstes ärztliches Wirken und Können setzt da ein, wo die Heilbarkeit der Krankheit aufhört. » ${ }^{22}$ Diese Worte haben heute, eine Generation später, eine noch größere Bedeutung als damals.

Klaesi, der eine internationale Praxis hatte, wollte nichts mit Krankenkassen oder Formularen zu tun haben; er ließ sich von seinen Patienten schriftlich von den entsprechenden Verpflichtungen entbinden. Gemäß den Gepflogenheiten vieler Hausärzte des letzten Jahrhunderts oder zu Beginn unseres Jahrhunderts stellte er armen Patienten keine oder nur sehr kleine Rechnungen aus ${ }^{26}$.

Zur Bedeutung, die dem Arzt bei einer Psychotherapie zukommt, sagt Klaesi : «Letzten Endes ist es doch bei allen psychotherapeutischen Anwendungen die Affektivität des Arztes, auf die es ankommt, ob es sich nun um Ergebnisse der sogenannten Tiefenforschung oder nur um eine einfache Bromverschreibung handle; stets wird die Geste noch wichtiger als der Inhalt. Selbstverständlich muß ein Patient nach allen Regeln der Wissenschaft gründlich (und zwar auch körperlich!) untersucht und die Therapie, wie schon erörtert, nach genauen Indikationen gewählt sein. Wenn diese aber in Anwendung kommt, ist es die Persönlichkeit des Arztes, welche die Hauptrolle spielt. »10

Klaesis Grundeinstellung und Haltung gegenüber jeglicher Psychotherapie soll durch nachstehende Sätze zusammengefaßt werden: «Ich habe auch keine Grenze gezogen zwischen der sogenannten kleinen und der groß einherschreitenden 'Tiefenpsychologie' - mit Absicht - denn wie sollte ich von einer Tiefenpsychologie sprechen, wenn mir Leib-Seele eine Einheit ist, tiefste, dem Bewußtsein verborgenste Gefühlsregungen und Triebanwandlungen sich durch die Affektphänomene an der Oberfläche zeigen und die führende und entscheidende oberste Instanz der Geist ist, zu dem man in überordnender Betrachtung, Versenkung und Andacht einkehrt, und wo wir auch ohne System immer willkommen sind! ${ }^{24}$ «Wem wissenschaftliche Grundlagen Geleise sind statt Wegweiser, ist ein Stümper; wer eine Methode hat und diese überall anwendet, ein Handwerker. Wer einem schizophrenen Tobsüchtigen, der sich mit dem Plan einer Erfindung, angeblich geeignet, jedes Flugzeug aus der Luft herunterzuholen, ins Kriegsministerium gedrängt hatte, dort vor die Türe gestellt und dann interniert wurde, nicht allen Ernstes den Dank des Vaterlandes auszusprechen sich genötigt fühlt dafür, daß er helfen wollte, obgleich der fragliche Apparat, wie der Kranke ja selbst wisse, vorläufig nur gut gemeinte Idee sei, versteht nicht, mit Herzensgüte, Schöpferwillen und Phantasie umzugehen. Wer nicht in jedem Kranken, auch im allereinfältigsten, den Mitbürger sieht und in der verwahrlostesten Patientin etwas von dem Fraulichen, ist als Seelenarzt fehl am Platz ... ${ }^{23}$

Die Ausdrucksphänomenologie kann an den Symptomen vorbeisehen und die wesentliche Frage aus irgendeinem Ausdrucksphänomen konstruieren. Klaesi berichtet folgendes Beispiel: 
Eine begüterte Patientin aus dem europäischen Adel wurde während zwölf Jahren von den verschiedensten Ärzten wegen Schlaflosigkeit erfolglos behandelt. Schließlich kam sie zu Klaesi. Das erste, was ihm auffiel, war, daß die attraktive Dame mit den teuersten Stoffen bekleidet war. Im scharfen Kontrast zu dieser eleganten Kleidung war ihre Kopfbedeckung, die furchtbar aussah und in keiner Weise zu der übrigen Erscheinung passen wollte. Klaesi fragte sie sofort: «Warum haben Sie einen so gräßlichen Hut auf?» Die Patientin erwiderte beleidigt : «Ich habe geglaubt, es hier mit einem ernsthaften Arzt zu tun zu haben.» Klaesi: «Natürlich, ein ernsthafter Arzt interessiert sich aber für alles.» Die Patientin beginnt darauf zu weinen, etwa 20 Minuten lang. Klaesi fragte sie, warum sie geweint habe. Antwort: «Weil es seit zwölf Jahren das erstemal ist, daß sich ein Mann nach meiner Garderobe erkundigt.» Klaesi: «Wie lange sind Sie verheiratet?» Die Patientin: «Zwölf Jahre.» Klaesis Antwort: «Dann muß ich die Sprechstunde hier abbrechen und Sie bitten, zusammen mit Ihrem Herrn Gemahl in vier Tagen, am Freitag, sich wieder hier einzufinden.» Er wollte also nicht, daß sie sich über ihren Mann beklagte, denn solches hätte die Entfremdung zu ihm gesteigert. Als jener Freitag da war und der Wagen vor dem Tor stand, entstieg ihm der Gemahl; er kam Klaesi mit offenen Armen entgegen. «Sie haben meine Frau gerettet!» sagte er ihm, umarmte ihn und erzählte lächelnd und doch zu Tränen gerührt, was geschehen war: «Statt vergrämt oder scheu oder mit schlechtem Gewissen wie bisher, wenn sie beim Arzt gewesen war, kam sie lebhaften Schrittes auf mich zu, fiel mir um den Hals und erzählte, tief ergriffen, wie die Sprechstunde verlaufen sei. Der Arzt vermied peinlichst, ihr Gelegenheit zu geben, sich über mich zu beklagen, wissend, daß sie dann mir gewiß nicht frei und zugetan wieder begegnen könnte. Sie war so ergriffen, daß ich mit ihr nicht im Speisesaal essen wollte, sondern vorschlug, die Mahlzeit in unserem Zimmer einzunehmen. Frei und doch bekümmert erwartend, machte sie erst nach jeder Aussage halt und bedachte ernst, was ich sagte; aber sichtlich wurde sie freier und freier.» Beim Schlafengehen verzichtete die Patientin auf das Schlafmittel, und mit Erfolg: «Wir redeten und redeten, weinten und lachten und weinten bis tief in die Nacht hinein.» Nach dieser zweiten Aussprache reiste das Paar in bestem Einvernehmen nach Hause. Wie Klaesi später hörte, blieb die Dame auch schlafmittelfrei ${ }^{28}$.

Mit Recht weist Theodor Spoerri auf die Gefahren einer falschen Anwendung der Ausdrucksphänomenologie hin. Er sagt, nachdem er Klaesi erwähnt hat: «Die Epigonen meinen, sie könnten es auch, ohne das entsprechende Niveau und die daraus folgende klinische Richtigkeit, oder: es wird eine Methode daraus gemacht, seziert, analysiert, und was bleibt, sind Leichen. ${ }^{29}$ 
Klaesi berichtet z.B., wie er einen erregten Patienten zur Ruhe bringen konnte:

Die Eltern eines Studenten, der als außergewöhnlich gefährlicher Angreifer und Zerstörer in einer Privatklinik hospitalisiert war, baten Klaesi um Hilfe. Dieser besuchte den Studenten und fand ihn, von zwei Pflegern bewacht, im Werg liegend, da er alles zerriß und alle angriff, wenn er nicht festgebunden war. Die Kollegen waren höchst verwundert, daß Klaesi sich entschloß, es mit ihm in Knonau zu versuchen, und ihn allein in seinem Wagen mitnahm. Er sah aber für Klaesi nicht aus wie ein Bösewicht, sondern wie ein Mißverstandener, und als Klaesi bemerkte, daß er bestimmte seiner Bewegungen nachahmte, hatte er keine Bedenken, ihn gleich mit sich zu nehmen ${ }^{28}$. Klaesi schildert diesen Fall wie folgt:

«Ich habe einen notorischen Zerstörer, der seine Eltern ein Heidengeld gekostet hatte, von einer Stunde zur anderen beruhigen und ihm die Zerstörungssucht abgewöhnen können, indem ich ihm ein Notizbüchlein überreichte mit den Worten, ich hätte gehört, daß er hie und da etwas zusammenschlage und zerreiße; es sei mir nicht gegeben, ihm eine Aufsicht zu bestellen, und ich fände solches auch überflüssig, weil ich ihn als Kavalier betrachte und ohne weiteres annehme, er sei so freundlich, selber Buch zu führen über alles, was er zerstöre, damit gewissenhaft Rechnung gestellt werden könne.» ${ }^{23}$ Das Notizbuch blieb unbeschrieben, weil er sich schon andern morgens dankbar zur Arbeit mit den übrigen Hausinsassen meldete ${ }^{28}$.

«Ich habe solche Beurteilung und Wertung der Symptome als Ausdrucksund Abwehrerscheinungen und als Hinweise auf die Charakteranlagen früher Aestimation genannt ... Heute rede ich von Eidodiagnostik und von der auf sie ausgerichteten Psychotherapie als von Eidodiathese» (Wiederherstellung des Urbildes) ${ }^{23}$.

Klaesi lehrte seine Schüler, jeden Kranken (nicht Krankheit!) individuell zu behandeln. Nicht auf das Gerede solle man sich verlassen, sondern auf das Verschwiegene und den «Ausdruckshader». Er verstand darunter die unterdrückten, nicht zum Ausdruck gebrachten Gemütsbewegungen. «Meine Schüler schrieben von 'Ausdrucksphänomenologie', ich von Aestimation und dann Eidodiathese. Heute bin ich belehrt, daß es Anti-Eidodiathese heißen muß, weil es um das im Bild Widersprüchliche geht oder um das, was darin fehlt, wenn es nicht von der Türschwelle gesagt wird, über welche der Eintretende stolperte. ${ }^{27}$

Bei Schizophrenen sind Tendenzen und Strebungen vorhanden, die dem Abkapselungsprozeß entgegenwirken. Klaesi nannte sie Selbstheilungstendenzen ${ }^{23,14}$; die aus ihnen hervorgehenden, in die Tat umsetzenden Willensäußerungen nannte er Selbstheilungsversuche ${ }^{23}$. Klaesi hält es für die Aufgabe jedes Psychothera- 
peuten, nach solchen Selbstheilungstendenzen zu suchen und diese als solche zu erkennen. Es könne dem Kranken dann geholfen werden, indem er belehrt werden muß, diese Tendenzen auf wirklichkeitsgerechte und bessere Weise zu erleben und zu verwirklichen. Die Querulanz kann z.B. einen Selbstheilungsversuch erster Ordnung darstellen, da sie in höchstem Maße geeignet ist, den Kontakt mit der Umwelt zu erhalten, wenn auch auf höchst ungeeignete Weise ${ }^{23}$.

Als Klaesi 1933 Direktor der Psychiatrischen Universitätsklinik Waldau in Bern wurde, entließ er so viele Kranke wie nur möglich. Ärzte und Mitglieder der Behörde waren bis anhin gewohnt, für einen Patienten, der in die Waldau eingewiesen werden sollte, kämpfen zu müssen, da die Waldau chronisch überfüllt war. Dies änderte sich schlagartig mit der Amtsübernahme von Klaesi, der bei solchen Anmeldungen ins Telephon zu rufen pflegte: «Lieber zwei statt einen. » ${ }^{31}$ Er wußte, «daß eine Frühentlassung manchem Kranken gut tut, auch wenn sie die Erfolgsstatistik der Anstalt verschlechtert, so entließ er und verursachte bei manchem Patienten, der zwar immer geschimpft und hinausgedrängt hatte, wehe Abschiedsschmerzen, wenn er nun die Waldau tatsächlich verlassen mußte ». ${ }^{31}$ Schon in Zürich wurde Klaesi von seinem Lehrer, Eugen Bleuler, zugezogen, um bei Platznot Entlassungen vorzunehmen ${ }^{28}$.

\section{Beiträge zu peripheren und nichtpsychiatrischen Gebieten}

Klaesi setzte sich für den «Hausarzt ältester Schule» ein. Seine diesbezüglichen Vorstellungen muten bemerkenswert modern an und könnten ebensogut in diesen Tagen geschrieben worden sein : «Um der Aufgabe genügen zu können, braucht er [gemeint ist der Hausarzt] allerdings einen umfassenderen psychiatrischen Unterricht, als ihn der Durchschnittsmediziner bisher genossen hat. Jener muß daher so gestaltet werden, daß der Student nicht nur psychiatrische Diagnosen machen, sondern vor allem psychiatrisch beobachten, denken und sprechen lernt. Neben der Klinik sollen propaedeutische Fächer, sollen auch psychologisches Denken in der Medizin, Psychotherapie, Kinderpsychiatrie usw. gelesen werden. » ${ }^{17}$

Deutlich nimmt Klaesi auch zur religiösen Frage innerhalb einer psychotherapeutischen Beziehung Stellung: "Wir kommen in priesterliche Bezirke. Ernst Kretschmer ist dagegen, daß der Arzt und Psychotherapeut sich in diese einmische. Sofern damit gesagt sein will, Dogmatik, Apologetik, Kasuistik und Absolution seien nicht seine Sache, bin ich einverstanden. Er darf auch keine Proselyten, und noch weniger Atheisten machen, aber für mich nehme ich das Recht in Anspruch, die religiösen Bindungen wieder zu knüpfen, wenn ihre Lockerung Schaden stiftet, und Gleichgültige und Abtrünnige zur Kirche zurückzuschicken, wenn sich der Verzicht auf deren Obhut als eine Kurzschlußhandlung 
erweist und in die Obdachlosigkeit geführt hat. Anläßlich einer klinischen Gastvorstellung in katholischem Kriegsland habe ich nach ausdrucksphänomenologischer Klärung der Beweggründe eines Selbstmordversuchs, und nachdem der Patient zum Leben zurückgefunden hatte und zur Entlassung vorgesehen war, als Protestant auf die unerläßliche Ergänzung der Psychotherapie durch den Priester hinweisen müssen, weil das Gesicht nicht gerettet und die Wiederherstellung nicht vollwertig sei, solange der Patient nicht auch die kirchliche Absolution habe; der Arzt müsse den Geistlichen über seinen Beruf aufklären, natürlich indem er sich jeglicher Wünsche bezüglich der Stellungnahme des Priesters enthalte. Ist der Heilbedürftige ein Protestant, erkühne ich mich, das Wort selbst auszulegen, weil ich wegen der Verschiedenheit der Richtungen im Protestantismus nicht wissen kann, in welcher Form und mit wieviel Sicherheit und Inbrunst es der Pfarrer tun würde, und weil es gerade auf diese jedes Mal sehr ankommt. ${ }^{24}$

Klaesi nimmt auch zu sozial-psychohygienischen Fragen Stellung: «Die Psychohygiene darf ihre Aufgabe nicht in der Wegbereitung im Sinne der Wegräumung möglichst aller Anstände und Hindernisse und in der Erziehung zu einem geruhsamen harmonischen Lebenswandel sehen, sondern, in Würdigung der lebensnotwendigen, kräftefördernden Beziehung zwischen Reiz und Reaktion, Anlauf und Abwehr in den Lehren von der 'Utilitas miseriae' und vom Glück der Unterstellung in eine leitende Idee, ob von innen, außen oder oben, sowie des leidenschaftlichen inbrünstigen Kampfes für dieselbe. Geben ist seliger als nehmen. Wenn Du zwei Röcke hast, so gib einen dem, der keinen hat. Unser Leben währet 70 Jahre und wenn es hochkommt, so sind's 80 Jahre und wenn's köstlich gewesen ist, so ist's Mühe und Arbeit gewesen - und nicht Pensionierung schon mit 50 oder $60 .{ }^{15}$

Klaesi verfaßte mehrere Dramen und Sonette. Zur Zeit schreibt er eine Tragödie mit dem Titel «Kain und Abel». Als Beispiel und Kostprobe sei aus dem Christusdrama «Gott und sein Zweifler» folgender Passus über das Volk zitiert:

«Volk! Fragt ihr nach dem Volk? Das wißt auch ihr:

Das Volk ist dümmer als das dümmste Tier.

Tier hat Gedächtnis, Haltung, Witterung.

Nur einmal schrecken Täuschung und Gefahr,

Beim zweiten stutzt schon stallverdummtes Schwein,

Doch Volk - das Volk - fällt immer neu herein.

Betrüge, lüge, stiehl soviel du kannst

Im Scheingefecht für Sicherheit und Frieden!

Versprich ein Dasein ohne Not hienieden!

Wieviel du auch für dich vorweggeklaut,

Volk schwelgt im Vorgefühl und glaubt und glaubt. ${ }^{25}$ 
Ernst Blum schreibt über Klaesis Dichtkunst: «In der Vielfalt der Themen, in denen Klaesi sich in seinen Sonetten zum gestaltenden Ausdruck bringt, finden wir keines, das sich mit seiner Einstellung zum Beruf und dessen mannigfachen und tiefen Problemen befaßt - großartig! So wenig ist ihm Psychiatrie 'Fach', isolierter Eigenstand, als daß er sie dichterisch auch nur mit einem Wort erwähnen müßte! ${ }^{2}$

Es ist das Verdienst Manfred Bleulers, nachgewiesen zu haben, was die endokrinologische Psychiatrie Jakob Klaesi verdankt. Bleuler schreibt: «In der endokrinologischen Psychiatrie haben wir die phänomenologische Betrachtung, wie sie Klaesi betreibt, keineswegs immer auszuschalten, sondern wir haben sie sehr oft nötig, um die Beziehung von Mensch zu Mensch mit dem Kranken herzustellen, um zu entdecken, wie ihm zumute ist und was er ist. ${ }^{1}$ Bleuler betont auch, daß ein Trennungsstrich zwischen endokrin bedingtem und persönlichkeitsbedingtem Aussehen nicht gerechtfertigt sei. Der durch seine endokrine Krankheit Entstellte empfindet dies subjektiv nicht immer als etwas Fremdes, sondern die Veränderung kann im Gegenteil als ein Ausdruck seines Ichs empfunden werden : «Der Myxödematöse kann manchmal seine unbeholfene Schwerfälligkeit, sein Wärmebedürfnis, seine Standortgebundenheit und Abhängigkeit als die Erfüllung dessen empfinden, was er ist und sein will. ${ }^{1}$ Bleuler erinnert auch daran, daß psychisch regressive Erscheinungen gleichartige in der endokrinen Sphäre zur Folge haben können, und nennt als Beispiel den Zusammenhang zwischen Amenorrhoe und seelischer Ablehnung des Frauwerdens. «Wenn Klaesi empirisch und intuitiv viel mehr Zusammenhänge zwischen Struktur und Triebhaftigkeit gefunden hat, als die meisten vor ihm je erahnt haben, so gibt ihm die heutige Endokrinologie nachträglich den physiologischen Nachweis, daß er auf konkreter Grundlage aufbaute. ${ }^{1}$

Klaesis Schüler schrieben zu seinem 70. Geburtstag von ihrem Lehrer: «Diese Tat [gemeint ist die Schlafkur mit Somnifen] leitet im eigentlichen Sinne die moderne Epoche der sog. somatischen Behandlungsmethoden der Schizophrenie ein und nimmt die heute allmählich sich allgemein durchsetzende Erkenntnis vorweg, daß jede körperliche Therapie dieser Krankheit nur eine Hilfe darstellt, den Schizophrenen der Psychotherapie, der Anpassung an die Umwelt zugänglich zu machen und aufzuschließen. Diese Konzeption konnte von Klaesi so früh bereits in ihrer vollen Bedeutung gefaßt werden infolge seiner, wie man wohl sagen kann, außerordentlich entwickelten und erstaunlichen künstlerisch intuitiven Gabe, sich in das Wesen ihm gegenüber stehender Kranker bis ins Letzte auf 
Grund ihres ausdrucksmäßigen Verhaltens einzufühlen, und so die Grundstruktur ihrer Persönlichkeit, die Motive ihres Handelns und den Sinn ihrer krankhaften Symptome zu durchschauen. Auf Grund dieser Gabe, die ihn zu einem der erfolgreichsten und bekanntesten aus aller Welt aufgesuchten Psychotherapeuten werden ließ, hat er seine Methode der phänomenologischen Ausdrucksanalyse entwickelt. ${ }^{5}$

Abschließend sei Theodor Spoerri, ebenfalls ein Schüler Klaesis, erwähnt, der zu Klaesis 90. Geburtstag schrieb: «Jakob Klaesi, selbst schon ein Stück Psychiatergeschichte, sieht sich der 90 jährige von Geschichten über seine mythenbildende Person umgeben, in denen er handgreiflich und augenfällig den Gesten, Worten und Handlungen ein Bewegungsdrama mit den menschlichen Verstrikkungen abliest und, das Menschliche in der Verstrickung betonend, die Verstrickung zum Fingerzeig auf die Lösung daraus, auf Ordnung und Aufgabe werden läßt. Er kann das. Er kann das für eine politische Kontroverse in den Vereinigten Nationen wie für den Ausdruckshader eines Zwiespältigen und die Zerfahrenheit eines Schizophrenen. ${ }^{30}$

\section{Diskussion}

Die Lehren und Anschauungen Klaesis in eine bestimmte Schule oder Richtung einzuordnen, ist kaum möglich. Henri Ellenberger ${ }^{4}$ weist auf die Ähnlichkeit mit Alfred Adler hin. Insbesondere wird die Erklärung der Neurose als Folge der «kratophoren» und «aristophoren» Triebe im Adlerschen Sinne verstanden. Klaesis Deutung des Ödipuskomplexes soll laut Ellenberger mit der Adlers identisch sein.

Gewisse Parallelen zu den Lehren Adlers dürften unbestreitbar sein. Trotzdem kann Klaesi unseres Erachtens nicht einfach als «Adlerianer» eingestuft werden. Der Ödipuskomplex z. B. spielte bei Adler eine untergeordnete Rolle. Er spielte nur insofern eine Rolle, als «verwöhnende Eltern die Neigung haben, das gegengeschlechtliche Kind besonders an sich zu ziehen, und damit möglicherweise sexuelle Empfindungen und Phantasien in diesem wachrufen. Die sexuellen Empfindungen wiederum können das primäre Minderwertigkeitsgefühl verstärken, da sie, von der Gesellschaft abgelehnt, im Kind sekundär Schuld und dementsprechend weitere Minderwertigkeitsgefühle erzeugen. ${ }^{32}$

Klaesi hat in seinem Schrifttum unseres Wissens den Ödipuskomplex nur einmal erwähnt, und zwar in folgendem Zusammenhang:

«Was wissen wir von einem Kind, das infolge starker aristophorer Begabung früh zu Gemeinschaft, Austausch und Überblick strebt, aber bei streitenden Eltern, welche das 
Gefühl von Geborgenheit und Pflege nie aufkommen lassen? Es findet Befriedigung, Annäherung und Bindung in Tagträumen, Phantastereien oder bei sexuellen Spielereien mit Gleichaltrigen und kann bei diesen bleiben, bis sich Konflikte melden. An mangelndem Widerhall aus der Erzieherschaft können auch ein frühwacher schöpferischer Kraftwille, Selbständigkeitsdrang und Mannesmut stranden. Schwer erziehbare, frühreife Knaben, die schon in ersten Jugendjahren mit dem Vater sich messen müssen, zanken und lassen sich von ihm vor die Türe stellen, nicht aus Eifersucht oder Protest nach dem Schema oben - unten, sondern aus Kraftgefühl. Sie wollen sich im Kampfe üben, und zwar mit dem Stärksten, nicht mit dem Kleineren, wollen ihre Selbständigkeit behaupten und Enttäuschungen und Beschimpfungen ertragen lernen, denn sie ahnen voraus, da $\beta$ diese dem Entschluß zur Männerrolle angetraut sind. Fehlt dann nur noch die Diagnose: 'Ödipuskomplex'. ${ }^{24}$

Zweifellos erinnern diese Zeilen an das Minderwertigkeitsgefühl und Machtstreben im Sinne Adlers. Die Einstellung Klaesis zum Ödipuskomplex wird jedoch nicht klar, sie wird nur in kritisch-fragendem oder gar ironischem Sinne angedeutet.

Wenn gewisse neurotische Symptome, wie z.B. das Bettnässen, für Adler lediglich der Ausdruck dafür sind, daß der Betreffende das Interesse der Umgebung auf sich lenken will ${ }^{32}$, so hat Klaesi dafür eine wesentlich differenziertere Erklärung. In diesem Zusammenhang sei an seinen Artikel «Über die psychogenen Ursachen der essentiellen Enuresis nocturna infantum» erinnert, in welchem er die Enuresis als Schuldersatzhandlung beschreibt ${ }^{9}$.

Ein einziges Mal geht Klaesi kurz auf eine wesentliche Anschauung Adlers ein und stellt sie derjenigen Freuds gegenüber: «Alle [gemeint sind Protesthandlungen und neurotische Symptome] geschehen 'als ob' von bestimmten Fiktionen aus und in Befolgung einer bestimmten Leitlinie. Da sie also zielgerichtet sind, sprach Adler von der finalen Orientiertheit im Gegensatz zur kausalen der Freudschen, vom Affekt betonten Erlebnissen abhängig gemachten. Der Unterschied fällt aber dahin, wenn man sich Rechenschaft gibt, daß die Symbolhandlungen nach Freud der Lustbefriedigung dienen und also auch zweckgerichtet sind. ${ }^{24}$

Daß Klaesi auch in der Auslegung von Träumen andere Wege als Freud ging und andere Ansichten vertrat, haben wir bereits gelesen. Der Traum als Gesamtes ist für Klaesi wesentlicher als die einzelnen darin vorkommenden Teile; dies stimmt zwar im großen und ganzen mit der Auffassung Adlers überein, darf jedoch nicht als für Adler spezifisch angesehen werden. In diesem Zusammenhang sei an das Traumverständnis von Medard Boss erinnert ${ }^{3}$.

Einflüsse von C.G.Jung und von A.Mäder werden bei Klaesi deutlich; z.T. werden die betreffenden Autoren auch namentlich erwähnt. Das Faktum, daß sich Klaesi intensiv mit Freud auseinandergesetzt hat, dürfte ebenfalls Spuren hinterlassen haben, obschon er sich Freud gegenüber kritisch-reserviert verhielt. Klaesis langjähriger Lehrer, Eugen Bleuler, setzte sich für die Lehren Freuds 
ein, auch wenn er, besonders in späteren Jahren, nicht in allen Punkten einigging. Auch die Phänomenologie von Karl Jaspers wird expressis verbis erwähnt. Klaesi betont aber, daß er, im Gegensatz zu Jaspers, nicht auf die offenbarten, sondern auf die unterdrückten Gemütsbewegungen abstellt ${ }^{27}$.

Klaesi maß der Erscheinung und Haltung des behandelnden Arztes eine wichtige Rolle bei. Diese spielten zu Beginn unseres Jahrhunderts im täglichen Leben eine größere Rolle als heute, wo Autoritäten nicht mehr gefragt sind, wo «autoritär» fast eine ausschließlich negative Bedeutung erhalten hat. Dennoch setzt man sich auch in neuerer Zeit mit diesem Problem auseinander; es sei in diesem Zusammenhang an das Stichwort «die Droge Arzt» von M. Balint erinnert.

Eine ganz andere Frage, die tatsächlich an Adler gemahnt, sei hier angeschnitten: Es ist diejenige der sogenannten Organminderwertigkeit, bzw. der Überkompensation im persönlichen Leben Klaesis. Wenn er auch nicht von Kind auf schwerhörig war, wurde er doch schon im jugendlichen Alter aufs schwerste durch die Krankheit behindert. Trotz, oder sogar wegen dieses Leidens entschied er sich innerhalb der Medizin für die Psychiatrie, wo das Gehör eine wesentliche Rolle spielt, sicher eine bedeutendere als z. B. in der Chirurgie, in der er anfänglich praktisch tätig war. Klaesi bewies aber in seinem Leben, daß der optischen Wahrnehmung, richtig verstanden und interpretiert, in der Psychiatrie eine fast ebenso wichtige Bedeutung zukommt. In diesem Sinne müssen auch seine phänomenologischen Ausführungen, die Lehre von den zum Ausdruck, bzw. nicht zum Ausdruck gebrachten Gemütsbewegungen, dem Ausdruckshader, verstanden werden.

\section{Zusammenfassung}

Der aus dem Glarnerland stammende, 1883 geborene Jakob Klaesi war bei Eugen Bleuler in Zürich Assistenzarzt und später Oberarzt. Von 1923 bis 1926 leitete er die neueröffnete Psychiatrische Poliklinik in Basel; gleichzeitig versah er den Oberarztposten in der damaligen Basler Heil- und Pflegeanstalt Friedmatt. Danach gründete Klaesi eine Privatklinik auf Schloß Knonau (Kanton Zürich). 1933 wurde er Ordinarius für Psychiatrie und Direktor der Waldau in Bern.

Klaesi war primär Psychotherapeut, stand den somatischen Behandlungsmethoden skeptisch gegenüber, obschon er zu Beginn der zwanziger Jahre in Zürich der Begründer der sogenannten Schlafkur mit Somnifen war.

Er verstand es, sich in das Wesen seiner Patienten und insbesondere in ihr ausdrucksmäßiges Verhalten zutiefst einzufühlen. Seine Einfühlungsgabe einerseits und seine philosophische Orientierung anderseits ermöglichten es ihm, die 
«phänomenologische Ausdrucksanalyse» zu entwickeln. Unterschiede und Parallelen zu den Lehren Freuds und Adlers werden erörtert.

\section{Literaturverzeichnis}

1. Bleuler, M.: Jakob Klaesi in der endokrinologischen Psychiatrie. Monatsschr. für Psych. u. Neurol. 125 (1953), Nr. 5/6, S. 310-319.

2. Blum, E.: Jakob Klaesi: Zum 70. Geburtstag am 29. Mai 1953. "Der kleine Bund» 104, Nr. 244, 1953.

3. Boss, M., "Es träumte mir vergangene Nacht, ...». Verlag Hans Huber, Bern 1975.

4. Ellenberger, H., Die Entdeckung des Unbewußten, Bd. 2. Hans Huber, Bern 1973.

5. Feremutsch, K., et al.: Zum Geleit., Jakob Klaesi zum 70. Geburtstag, gewidmet von seinen Freunden, Mitarbeitern und Schülern. Monatsschr. für Psych. u. Neurol. 125 (1953), Nr. 5/6.

6. Freud, S., Traumdeutung. Franz Deuticke, Wien 1950.

7. Haenel, Th., Zur Geschichte der Psychiatrischen Poliklinik Basel. Schw. Arch. Neurol. Psychiat. 124 (1979), S. 335-350.

8. Klaesi, J., Über das Psychogalvanische Phänomen, Med. Diss. Zürich/Leipzig 1912.

9. Klaesi, J.: Die psychogenen Ursachen der essentiellen Enuresis nocturna infantum. Schw. Arch. Neurol. Psychiat. 35 (1917), S. $371 \mathrm{ff}$.

10. Klaesi, J.: Über psychiatrisch-poliklinische Behandlungsmethoden. Zschr. der ges. Neurol. Psychiat. 36 (1917), S. 431-450.

11. Klaesi, J., Über das Wesen und die Bedeutung der Stereotypien. Monogr. als Beiheft zur Mschr. Psychiat. Neurol. 1920 (Habilitationsschrift).

12. Klaesi, J.: Über Somnifen, eine medikamentöse Therapie schizophrener Aufregungszustände. Schw. Arch. Neurol. Psychiat. 8 (1921), S. 131.

13. Klaesi, J.: Über die therapeutische Anwendung der «Dauernarkose» mittels Somnifens bei Schizophrenen. Zschr. der ges. Neurol. Psychiat.74 (1922), S. 557.

14. Klaesi, J.: Einiges über Schizophreniebehandlung. Zschr. der ges. Neurol. Psychiat. 78 (1922), S. 606.

15. Klaesi, J.: Hygiene der Hygiene. Schweiz. Med. Wschr. 65 (1935), Nr. 24, S. 563-565.

16. Klaesi, J.: Durchgangsklinik oder nicht? Z. Psychiat. 103 (1935), S. 330.

17. Klaesi, J.: Über geistige Hygiene. Vortrag, gehalten auf Einladung der Herbstversammlung der Schweiz.Vereinigung für Psychiatrie, Bern 1934, in: Vom seelischen Kranksein, Vorbeugen und Heilen. Paul Haupt, Bern/Leipzig 1937.

18. Klaesi, J.: Die Irrenanstalt als Weg zur Rückkehr ins Leben. Vortrag, gehalten im Hilfsverein für Geisteskranke der Stadt Bern, Winter 1935/36, in: Vom seelischen Kranksein, Vorbeugen und Heilen. Paul Haupt, Bern/Leipzig 1937.

19. Klaesi, J., Vom seelischen Kranksein, Vorbeugen und Heilen. Paul Haupt, Bern/Leipzig 1937.

20. Klaesi, J.: Ohne Insulin. Schweiz.Med.Wschr. 68 (1938), Nr. 42, S. 1164f.

21. Klaesi, J.: Zur somatischen Therapie der Schizophrenie. Schweiz. Med.Jahrbuch, 1942.

22. Klaesi, J., Der unheilbare Kranke und seine Behandlung. Rektoratsrede Universität Bern, 18.11.1950. Paul Haupt, Bern 1950. 
23. Klaesi, J.: Psychotherapie in der Klinik. Mschr. Psychiat. Neurol. 124 (1952), S. 334353.

24. Klaesi, J.: Über Neurosenlehre und Psychotherapie. In: Handbuch der Inneren Medizin, herausgegeben von Gustav v. Bergmann, 4. Auflage. Springer, Berlin/Göttingen/ Heidelberg 1953.

25. Klaesi, J., Gott und sein Zweifler. Werner Classen, Zürich 1969.

26. Klaesi, J.: Persönliche Mitteilung vom 12.2.1975.

27. Klaesi, J.: Pongratz, L. J., Psychiatrie in Selbstdarstellungen. Hans Huber, Bern 1977.

28. Klaesi, J.: Persönliche Mitteilungen vom 3.2., 23.3. und 2.4.1979.

29. Spoerri, Th., Klinik und Ausdrucksphänomenologie: Aktuelle Fragen der Psychiatrie und Neurologie, Vol. I. Karger, Basel 1964, S. 185-196.

30. Spoerri, Th.: In: Pongratz L. J., Psychiatrie in Selbstdarstellungen: Jakob Klaesi. Hans Huber, Bern 1977.

31. Wyrsch, J., 100 Jahre Waldau. Hans Huber, Bern/Stuttgart 1955.

32. Wyß, D., Die tiefenpsychologischen Schulen von den Anfängen bis zur Gegenwart. Vandenhoeck und Ruprecht, Göttingen 1966.

\section{Summary}

Jacob Klaesi, grown up in the region of Glarus and born in 1883, worked first as assistant and later as head-physician in Zurich in the Psychiatric University Hospital, directed by Eugen Bleuler. From 1923 to 1926 Klaesi became head of the new Psychiatric Out-Patient Clinic in Basel. At the same time, he was head-physician in the Psychiatric University Hospital (Friedmatt). Later on Klaesi founded the Private Clinic "Schloß Knonau» (Canton Zurich). In 1933 he became Chairman of Psychiatry and Director of the Psychiatric University Hospital in Berne (Waldau).

Klaesi considered himself primarily as psychotherapist, his attitude towards the methods of somatic treatments was a sceptical one, although he was in the early twenties in Zurich the founder of the psychiatric sleeping cure with Somnifene. Klaesi had a great empathy for his patients, understanding especially their expressive behavior. His capacity for empathy on one side and his philosophic orientation on the other side enabled him to develop his "phenomenological analysis of expression". Differences and parallels to the theories of Freud and Adler are discussed.

Dr. Thomas Haenel

Psychiatrische Universitätspoliklinik

(Chefarzt: Prof. Dr. Raymond Battegay)

Petersgraben 4

4031 Basel 